TITLE:

\title{
Exciton fine structure in a single carbon nanotube revealed through spectral diffusion
}

\section{AUTHOR(S):}

Matsuda, Kazunari; Inoue, Tadashi; Murakami, Yoichi; Maruyama, Shigeo; Kanemitsu, Yoshihiko

\section{CITATION:}

Matsuda, Kazunari ...[et al]. Exciton fine structure in a single carbon nanotube revealed through spectral diffusion. Physical Review B 2008, 77(19): 193405.

ISSUE DATE:

2008-05

URL:

http://hdl.handle.net/2433/87352

RIGHT:

c 2008 The American Physical Society 


\title{
Exciton fine structure in a single carbon nanotube revealed through spectral diffusion
}

\author{
Kazunari Matsuda, ${ }^{1, *}$ Tadashi Inoue, ${ }^{1}$ Yoichi Murakami, ${ }^{2}$ Shigeo Maruyama, ${ }^{2}$ and Yoshihiko Kanemitsu ${ }^{1,3, \dagger}$ \\ ${ }^{1}$ Institute for Chemical Research, Kyoto University, Uji, Kyoto 611-0011, Japan \\ ${ }^{2}$ Department of Mechanical Engineering, The University of Tokyo, 7-3-1 Hongo, Bunkyo-ku, Tokyo 113-8656, Japan \\ ${ }^{3}$ Photonics and Electronics Science and Engineering Center, Kyoto University, Kyoto 615-8510, Japan
}

(Received 9 April 2008; published 20 May 2008)

\begin{abstract}
We studied the spectral diffusion and exciton fine structures of single-walled carbon nanotubes (SWNTs) by using single carbon nanotube spectroscopy. Two types of single nanotube photoluminescence (PL) spectra were observed at temperatures below $50 \mathrm{~K}$, which are double peaks with spectral diffusion and a single peak without spectral diffusion. The spectral diffusion that occurs in the lower energy peak of the split PL spectrum is associated with the quantum-confined Stark effect. Our observations suggest that symmetry breaking caused by the strong local electric field enables us to observe the dark exciton state below the bright exciton state in single SWNTs.
\end{abstract}

DOI: 10.1103/PhysRevB.77.193405

PACS number(s): 78.67.Ch, 78.55.-m

Recently, the electronic properties of a single-walled carbon nanotube (SWNT) ${ }^{1}$ have attracted a great deal of attention because the Coulomb interaction in the quasi-onedimensional (1D) system is strongly enhanced, ${ }^{2}$ which leads to the formation of excitons with extremely large binding energies in the range of $200-400 \mathrm{meV}^{3,4}$ The exciton states of a semiconducting SWNT have complicated fine structures around the lowest energy due to the degeneracy of $k$ space. ${ }^{5-8}$ The electron and hole spin together with two degenerate $K$ and $K^{\prime}$ valleys lead to multiple exciton states with odd and even parity spin singlets. In a perfect SWNT, the optical transition from the exciton state with an odd parity to the ground state is dipole allowed (bright), while the other transitions are optically forbidden (dark). Theoretical calculations predict an energy separation of several $\mathrm{meV}$ between odd (bright) and even (dark) singlet states and a mixing of the two eigenstates when the crystal symmetry in a SWNT is broken. ${ }^{7}$ The exciton fine structures including the energy separation should be elucidated because the exciton fine structures strongly affect the optical properties of SWNTs, such as the photoluminescence (PL) quantum efficiency.

Progress in single object (molecule, quantum dot, or nanotube) spectroscopy has allowed the discovery of inherent optical and electronic properties beyond ensembleaveraged properties. ${ }^{9-17}$ A slight energy level fluctuation can be sensitively monitored through spectral jittering, i.e., spectral diffusion using single object spectroscopy. ${ }^{9-12}$ PL blinking and spectral diffusion have frequently been observed in semiconductor nanocrystals (quantum dots) because zerodimensional (0D) exciton states have discrete energy structures plus a large surface-to-volume ratio of nanocrystals is easily affected by external perturbations. Spectral diffusion has also been observed in single SWNTs, ${ }^{15-18}$ although details of the mechanisms, including an explanation of the spectral diffusion in quasi-1D exciton systems, are not clear. It is possible that the external perturbation causing spectral diffusion also induces symmetry breaking, thereby modulating the electronic state. Therefore, clarifying the spectral diffusion phenomena revealed by single nanotube PL spectroscopy provides new insight into exciton fine structures in SWNTs.
In this Brief Report, we report the exciton fine structures in SWNTs that were determined by using single nanotube PL spectroscopy. We found that SWNTs could be classified into two types based on single SWNT PL spectra at temperatures below $50 \mathrm{~K}$ : those with a narrow PL peak with an almost Lorentzian line shape and those with double PL peaks accompanying spectral diffusion. The exciton fine structures are discussed based on the characteristics of spectral diffusion.

The samples used in this study were spatially isolated SWNTs that were synthesized on Si substrates by using an alcohol catalytic chemical vapor deposition method. ${ }^{19}$ The Si substrate was patterned with parallel grooves, which were typically $\sim 1 \mu \mathrm{m}$ in width and $500 \mathrm{~nm}$ in depth, by using electron-beam lithography. The isolated SWNTs grew from one side toward the opposite side of the groove. Different samples were prepared by changing the growth temperature and time. Single nanotube PL spectroscopy was performed on samples grown at $750{ }^{\circ} \mathrm{C}$ for $30 \mathrm{~s}$ with a typical average luminescent SWNT number density of $\sim 0.1 \mu \mathrm{m}^{-2}$.

Single SWNT PL was performed at temperatures ranging from 4.7 to $100 \mathrm{~K}$ by using a custom-built confocal microscope. ${ }^{20}$ The SWNT samples were mounted in a cryostat and excited with a He-Ne laser $(1.96 \mathrm{eV})$. The laser light was focused on the sample surface through a microscope objective. The PL signal from the SWNT was detected by using a $30 \mathrm{~cm}$ spectrometer equipped with a liquidnitrogen-cooled InGaAs photodiode array.

Figure 1(a) shows the normalized PL spectra of two typical single SWNTs at $40 \mathrm{~K}$. We classified SWNTs into two types, a single peak without spectral diffusion (type A) and double PL peaks with spectral diffusion (type B) based on many single-nanotube PL spectra. Both SWNTs in Fig. 1(a) show the same chiral index of $(10,3)$, which was based on the emission energies given in Refs. 21 and 22. The PL peak energy changes between each single nanotube with the same chiral index due to the difference in the local environment and so on. The emission energies of type $\mathrm{A}$ and type $\mathrm{B}$ are almost the same at higher temperature. The spectral line shape in type A shows a good fit to a single Lorentzian function, as indicated by the solid line in Fig. 1(a). Figure 1(b) shows the contour plot of the temporal evolution of the 
(a)

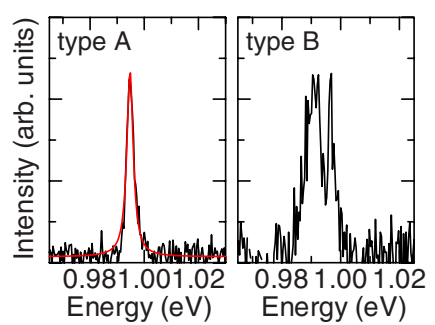

(b)

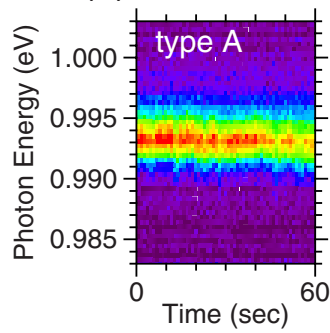

(c)

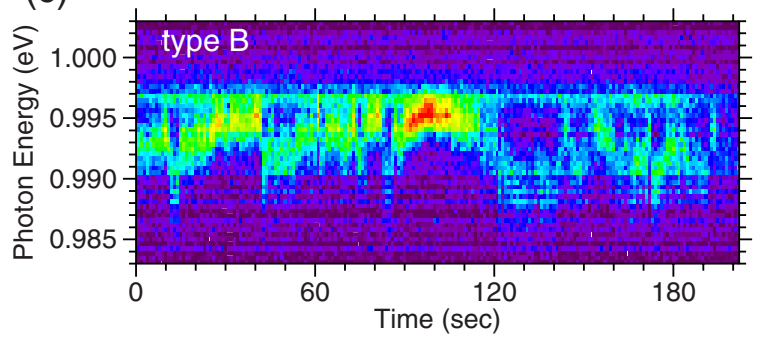

FIG. 1. (Color online) (a) Normalized PL spectra of typical single type A and type B SWNTs on the same sample at $40 \mathrm{~K}$ excited with He-Ne laser light. The solid line indicates the results of fitting analysis assuming a Lorentzian function. The temporal evolution of the PL spectrum of (b) type A and (c) type B SWNTs.

PL spectrum with an accumulation time of $1 \mathrm{~s}$. The peak position does not change for the observation time scale $(>1 \mathrm{~s})$ under continuous wave (cw) laser excitation conditions, as is expected from the exciton lifetime of about 30 ps. ${ }^{23}$ The PL properties of type A SWNTs are stable from 4.4 to $90 \mathrm{~K}$ (not shown here). These results indicate that the PL peak in a type A SWNT can be attributed to the recombination of a quasi-1D exciton in the bright exciton state.

The PL spectrum of a type B SWNT has double peaks, as shown in Fig. 1(a), which is in contrast to the single peak of a type A SWNT. The energy difference between the higher and lower energy peaks is estimated to be on the order of several meV, as discussed below in detail. The two peaks do not simply come from two spatially isolated SWNTs in the same excitation spot of $\sim \mu \mathrm{m}^{2}$, because the density of luminescent SWNTs in this sample was kept below $\sim 0.1 \mu \mathrm{m}^{2}$. Figure 1(c) shows the temporal evolution of the PL spectra of a type B SWNT. Note that the PL peak positions change over several seconds, even under $\mathrm{cw}$ laser excitation conditions, which is in contrast to the behavior of type A SWNTs [see Fig. 1(b)].

A PL peak fluctuation on the order of several seconds is observed, which is attributed to spectral diffusion (or spectral wandering). Figure 2 shows the temperature dependence of the temporal evolution of the PL spectrum of a type B SWNT. The PL peak positions do not change with time at temperatures above $70 \mathrm{~K}$, while there is an obvious PL fluctuation due to spectral diffusion that is clearly observed at temperatures below $50 \mathrm{~K}$. Note that there is a strong correlation between the SWNT type and the spectral diffusion, i.e., the spectral diffusion occurs with type B SWNTs, while distinct spectral diffusion is not observed with type A SWNTs. As the spectral diffusion strongly depends on the temperature and type of SWNT for the same sample, this

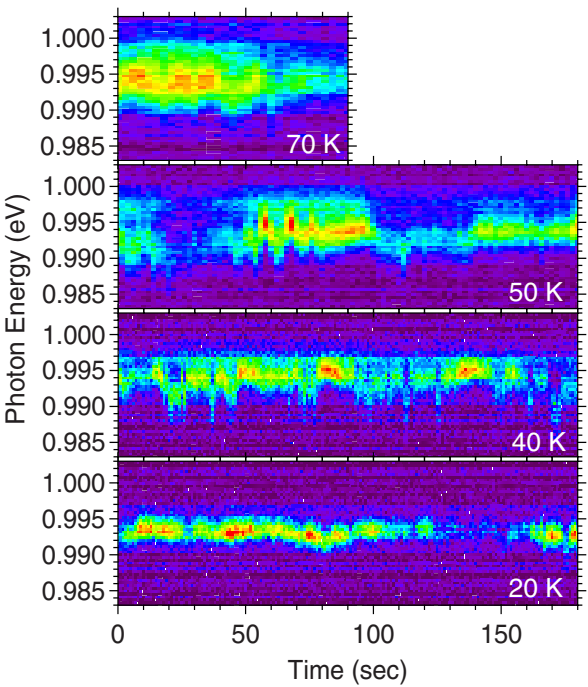

FIG. 2. (Color online) Contour plot of the temporal traces of a single nanotube PL spectrum (type B) from 10 to $70 \mathrm{~K}$ [assigned chiral index: $(10,3)]$. The detector accumulation time was $3 \mathrm{~s}$ at 70 $\mathrm{K}$ and $1 \mathrm{~s}$ at temperatures below $50 \mathrm{~K}$.

spectral diffusion cannot be explained by laser heating effects.

Figure 3 shows the temporal evolution of the PL spectrum of a type B single SWNT at $40 \mathrm{~K}$. The PL spectra clearly show two peaks [see also Fig. 1(a)], which are fit by lower energy side Gaussian and higher energy side Lorentzian functions (solid lines) at all times. We obtained the peak positions and linewidths of both peaks of the PL spectra.

Figure 4(a) shows the higher and lower PL peak energies that were obtained by the analysis as shown in Fig. 3. Although the energy difference of several $\mathrm{meV}$ between the

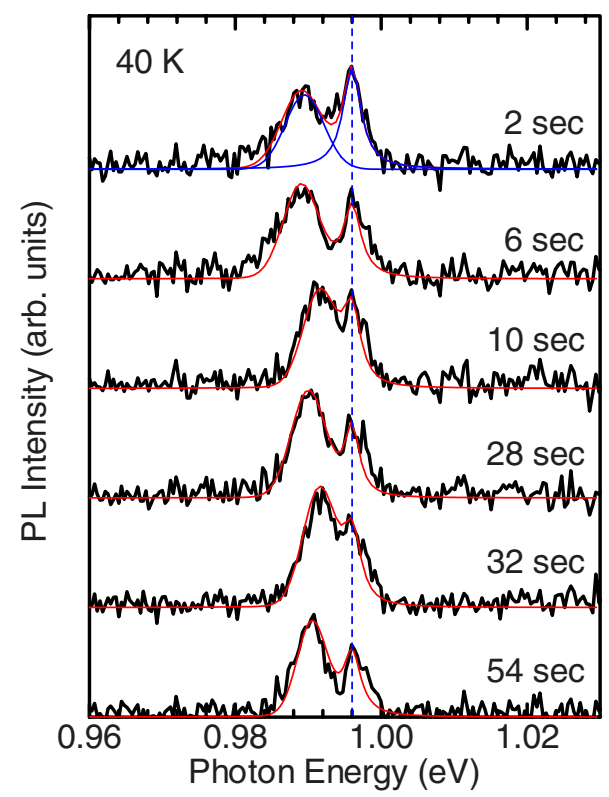

FIG. 3. (Color online) Temporal evolutions of the PL spectrum of a single type B SWNT at $40 \mathrm{~K}$. The solid curves indicate the results of fitting analysis assuming Gaussian and Lorentzian functions. 
(a)

(b)

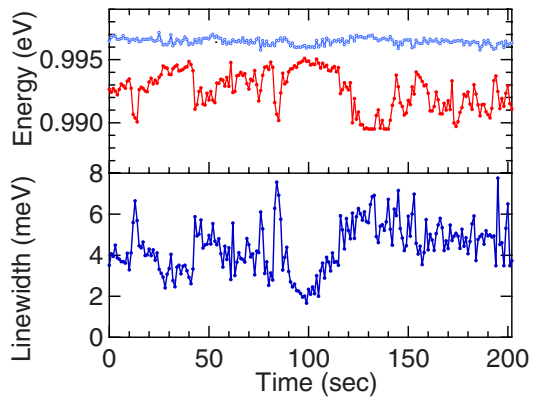

(c)

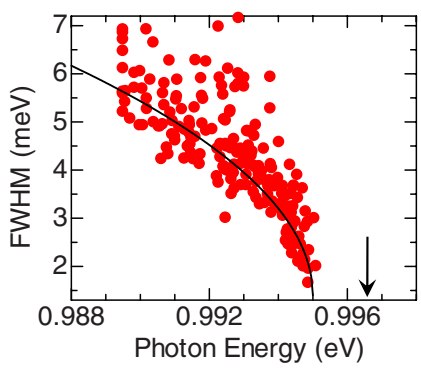

FIG. 4. (Color online) (a) Temporal trace of the higher and lower energy peaks of the PL spectra of a single type B SWNT at $40 \mathrm{~K}$. (b) The temporal trace of the linewidth (FWHM) of the lower energy peaks. (c) The PL linewidth (FWHM) as a function of the PL energy. The solid line corresponds to the square root energy dependence that was predicted from the quantum-confined Stark shift by using Eq. (2). The arrow indicates the energy position of the higher peak.

two peaks changes at times due to spectral diffusion, the energies of the higher energy peak do not change and only the lower energy peak fluctuates. Figure 4(b) shows the temporal evolution of the PL linewidth (full width at half maximum: FWHM) of the lower energy peak, which is based on the temporal evolution of about 200 spectra. The linewidth fluctuates over time. These results indicate that the spectral diffusion is associated with the energy and linewidth fluctuation of the lower PL peak in type B SWNTs. Figure 4(c) shows the PL linewidth (FWHM) plots of the lower energy peaks of a type B single SWNT as a function of the emission energy. The arrow indicates the position of the higher energy peak that corresponds to exciton recombination $(0.9965 \mathrm{eV})$ in the bright state. Note the finite energy gap of about 1.5 $\mathrm{meV}$ between the highest energy edge of the lower PL peak and the bright exciton energy. There is also a strong correlation between the linewidth and the emission energy, i.e., the linewidth sublinearly increases with the emission energy.

Let us discuss the spectral diffusion in SWNTs at low temperatures. Spectral diffusion has been inherently observed in 0D systems of single dye molecules ${ }^{9}$ and colloidal and compound semiconductor quantum dots ${ }^{10-12}$ because these spatially confined electronic states are easily affected by local external perturbations such as localized charges in the trap states. In contrast, the exciton states in quasi-1D SWNTs extend along a nanotube axis, and the exciton motion has a degree of freedom along the nanotube axis. Thus, local external perturbation should have little effect on the quasi-1D exciton states. In fact, the PL spectrum of a com- pound semiconductor quantum wire does not show spectral diffusion. ${ }^{24}$ Therefore, the spectral diffusion can be used as a measure of the extension of the exciton wave function. The two types of PL spectra from type A and B nanotubes with and without spectral diffusion imply that the difference arises from the extension of the exciton states.

The spectral diffusion can be explained by the fluctuation of localized charges. Localized surface charges in a SWNT in trap states, which are doped by impurities or defects, generate a strong local electric field, which causes a redshift in the exciton energy. This shift, $\Delta E$, is proportional to the square of the local electric field $F$ arising from the quantumconfined Stark effect, ${ }^{10,11}$

$$
\Delta E \propto F^{2} .
$$

The observed large spectral shift (diffusion) of several meV can be explained by a variation in $F$. Furthermore, a small, fast, local electric field fluctuation, $\delta F$, which possibly results from surface charge oscillations between trap states, ${ }^{10,11}$ causes small spectral shifts $[\Delta E(F+\delta F)-\Delta E(F)]$ and $F$-dependent PL line broadening. Therefore, the total width of the spectral using Eq. (1) is dependent on the spectrum shifts,

$$
\Delta E(F+\delta F)-\Delta E(F) \propto F \propto \sqrt{\Delta E} .
$$

In Fig. 4(c), we plotted the predicted PL line broadening by using Eq. (2). The experimental results demonstrate the inherent square root dependence that was predicted from the quantum-confined Stark effect. Therefore, the spectral diffusion of SWNTs is associated with the quantum-confined Stark effect and a strong local electric field is unintentionally applied to a type B SWNT.

Theoretical calculations predict that the exciton states around the lowest energy split into even (dark) and odd (bright) spatial symmetry states. ${ }^{5-8}$ The efficient PL occurs only from the bright state of exciton, because the other states are perfectly dark in an ideal SWNT in which nothing disrupts the spatial symmetry. Therefore, the PL from type A SWNTs comes from the recombination of quasi-1D excitons in ideal SWNTs. Conversely, Perebeinos et al. ${ }^{7}$ predicted that the wave function mixing redistributes between two states in a locally disordered SWNT because of symmetry breaking. Such mixing due to the symmetry breaking provides a finite oscillator strength in the dark state, i.e., a partially dark state, ${ }^{25,26}$ which might lead to light emission from the lower energy dark exciton. In this case, the two emission peaks from the bright and partially dark exciton states can be observed at lower temperatures at which the thermal energy is less than the splitting energy of the two states. The strong local electric field that gives rise to the spectral diffusion also breaks the symmetry of the SWNTs ${ }^{27,28}$ and to energy shift of exciton state due to the Stark effect. Therefore, the lower exciton PL peaks experimentally observed with the accompanying spectral diffusion from a type B SWNT [see Fig. 1(a)] are attributed to the recombination of the excitons in the partially dark state in the SWNT. The PL intensity and its energy dependence on the dark exciton might provide important information, however, at present it is difficult to understand these quantitatively. Here, we focus on the energy 
separation between the bright and dark exciton. The energy separation between two PL peaks during the spectral diffusion (e.g., about $6 \mathrm{meV}$ in the top panel of Fig. 3) is determined by both the energy shift due to the Stark effect and bright and dark exciton splitting. The estimated energy gap of $1.5 \mathrm{meV}$ in SWNTs with $d=0.94 \mathrm{~nm}$ [see Fig. 4(c)] corresponds to the splitting energy of the bright and dark exciton states, which is consistent with the theoretical calculation $^{7}$ and the experimental result that was estimated from the temperature dependence of the PL intensity behavior. ${ }^{26}$

In conclusion, we studied exciton fine structures by using single nanotube PL spectroscopy. We separated SWNTs into two types based on single nanotube PL spectra obtained at temperatures below $50 \mathrm{~K}$; a single peak without spectral diffusion (type A) and double PL peaks with spectral diffusion (type B). The single-peak PL spectrum arises from quasi-1D exciton recombination in the bright exciton state. It was found that the spectral diffusion occurs in the lower energy peak of the type B SWNT PL spectrum, which is associated with the quantum-confined Stark effect. Our observations show that the energy splitting between the bright and the dark exciton states is estimated to be about $1.5 \mathrm{meV}$ for $d=0.94 \mathrm{~nm}$ SWNTs.

The authors thank S. Kasai and T. Ono for support in fabricating the grooved $\mathrm{Si}$ substrate that was used to grow SWNTs. Part of the work conducted at Kyoto University was supported by a Grant-in-Aid for Scientific Research from JSPS (KAKENHI No. 17684012) and the Foundation for C\&C Promotion.

\footnotetext{
*matsuda@scl.kyoto-u.ac.jp

${ }^{\dagger}$ kanemitu@scl.kyoto-u.ac.jp

${ }^{1}$ S. Iijima, Nature (London) 354, 56 (1991).

${ }^{2}$ T. Ogawa and T. Takagahara, Phys. Rev. B 43, 14325 (1991); T. Ogawa, in Optical Properties of Low-Dimensional Materials, edited by T. Ogawa and Y. Kanemitsu (World Scientific, Singapore, 1995), p. 1.

${ }^{3}$ F. Wang, G. Dukovic, L. E. Brus, and T. F. Heinz, Science 308, 838 (2005).

${ }^{4}$ T. Ando, J. Phys. Soc. Jpn. 66, 1066 (1997).

${ }^{5}$ C. D. Spataru, S. Ismail-Beigi, L. X. Benedict, and S. G. Louie, Phys. Rev. Lett. 92, 077402 (2004).

${ }^{6}$ H. Zhao and S. Mazumdar, Phys. Rev. Lett. 93, 157402 (2004).

${ }^{7}$ V. Perebeinos, J. Tersoff, and P. Avouris, Nano Lett. 5, 2495 (2005).

${ }^{8}$ T. Ando, J. Phys. Soc. Jpn. 75, 024707 (2006).

${ }^{9}$ R. M. Dickson, A. B. Cubitt, R. Y. Tsien, and W. E. Moerner, Nature (London) 388, 355 (1997).

${ }^{10}$ S. A. Empedocles and M. G. Bawendi, Science 278, 2114 (1997).

${ }^{11}$ J. Müller, J. M. Lupton, A. L. Rogach, J. Feldmann, D. V. Talapin, and H. Weller, Phys. Rev. Lett. 93, 167402 (2004).

${ }^{12}$ M. Sugisaki, H.-W. Ren, K. Nishi, and Y. Masumoto, Phys. Rev. Lett. 86, 4883 (2001).

${ }^{13}$ A. Hartschuh, H. N. Pedrosa, L. Novotny, and T. D. Krauss, Science 301, 1354 (2003).

${ }^{14}$ J. Lefebvre, J. M. Fraser, P. Finnie, and Y. Homma, Phys. Rev. B 69, 075403 (2004).

${ }^{15}$ H. Htoon, M. J. O'Connell, P. J. Cox, S. K. Doorn, and V. I.
}

Klimov, Phys. Rev. Lett. 93, 027401 (2004).

${ }^{16}$ A. Hartschuh, H. N. Pedrosa, J. Peterson, L. Huang, P. Anger, H. Qian, A. J. Meixner, M. Steiner, L. Novotny, and T. D. Krauss, ChemPhysChem 6, 577 (2005).

${ }^{17}$ O. Kiowski, S. Lebedkin, F. Hennrich, and M. M. Kappes, Phys. Rev. B 76, 075422 (2007).

${ }^{18}$ K. Matsuda, Y. Kanemitsu, K. Irie, T. Saiki, T. Someya, Y. Miyauchi, and S. Maruyama, Appl. Phys. Lett. 86, 123116 (2005).

${ }^{19}$ S. Maruyama, R. Kojima, Y. Miyauchi, S. Chiashi, and M. Kohno, Chem. Phys. Lett. 360, 229 (2002).

${ }^{20}$ T. Inoue, K. Matsuda, Y. Murakami, S. Maruyama, and Y. Kanemitsu, Phys. Rev. B 73, 233401 (2006).

${ }^{21}$ S. M. Bachilo, M. S. Strano, C. Kittrell, R. H. Hauge, R. E. Smalley, and R. B. Weisman, Science 298, 2361 (2002).

${ }^{22}$ J. Lefebvre, J. M. Fraser, Y. Homma, and P. Finnie, Appl. Phys. A: Mater. Sci. Process. 78, 1107 (2004).

${ }^{23}$ H. Hirori, K. Matsuda, Y. Miyauchi, S. Maruyama, and Y. Kanemitsu, Phys. Rev. Lett. 97, 257401 (2006).

${ }^{24}$ M. Yoshita, Y. Hayamizu, H. Akiyama, L. N. Pfeiffer, and K. W. West, Phys. Rev. B 74, 165332 (2006).

${ }^{25}$ J. Shaver, J. Kono, O. Portugall, V. Krstic, G. L. J. A. Rikken, Y. Miyauchi, S. Maruyama, and V. Perebeinos, Nano Lett. 7, 1851 (2007).

${ }^{26}$ I. B. Mortimer and R. J. Nicholas, Phys. Rev. Lett. 98, 027404 (2007).

${ }^{27}$ V. Perebeinos and P. Avouris, Nano Lett. 7, 609 (2007).

${ }^{28}$ H. Zhao and S. Mazumdar, Phys. Rev. Lett. 98, 166805 (2007). 